\title{
Extending public insurance postpartum coverage: implications for maternal and child health
}

\author{
Jean L. Raphael ${ }^{1 凶}$ and Mona Patel ${ }^{2}$ \\ (c) The Author(s), under exclusive licence to the International Pediatric Research Foundation, Inc 2022
}

Pediatric Research (2022) 91:725-726; https://doi.org/10.1038/s41390-022-01949-5

The postpartum period represents a major state of medical and social vulnerability for women as they navigate both physiologic and psychological changes. ${ }^{1}$ The competing priorities of managing their own health in addition to caring for a newborn may have a compounding effect on mental health, manifested as depression or anxiety. Previous studies have demonstrated that poor maternal mental health adversely impacts maternal-infant bonding. ${ }^{2}$ During the coronavirus disease 2019 (COVID-19) pandemic, postpartum women experience challenges that may put them at elevated risk for mental health problems., ${ }^{3,4}$ The added challenges of the pandemic have heightened concerns about the potential deleterious effects on maternal-infant bonding. The study by Liu et al. contributes to the existing evidence by exploring relationships between mental health symptoms, psychological experiences, COVID-19-related concerns, and reported maternal-infant bonding experiences among postpartum women. ${ }^{5}$ The authors conducted a cross-sectional study of an online survey from the PEACE (Perinatal Experiences and COVID-19 Effects) Study. The primary dependent variable was maternal-infant bonding and independent variables consisted of COVID-19-related health, COVID-19-related grief, depression symptoms, anxiety symptoms, maternal self-efficacy, and social support. Consistent with the existing literature, the study found that postpartum women's depressive symptoms were associated with lower quality maternal-infant bonding. Maternal self-efficacy was positively related to maternal-infant bonding. COVID-19-related grief was significantly associated with lower bonding. However, COVID-19-related worries were associated with higher levels of maternal-infant bonding. The findings from this study are noteworthy in that they provide greater understanding of how the pandemic has impacted postpartum women, and consequently, maternal-infant bonding.

Liu et al. are to be commended for conducting this clinically relevant study and giving insights to a complex set of findings that have substantial implications for child neurodevelopment and the overall intergenerational health of families during the pandemic. In addition to contextualizing their results within the literature on maternal-infant bonding, the authors also provide key considerations for health care providers and areas for further study. They highlight (1) screening for postpartum depression during maternal follow-up visits and well-child visits; (2) focusing on dyadic interventions that increase maternal self-efficacy; and (3) assessing the unique COVID-19-related health and grief concerns of families. While the authors articulate an evidence-informed template for future clinical and research investments to transform practice, they do not address the critical role of policy in ameliorating postpartum psychological risks to mother-infant bonding. In this commentary, we focus on the expansion of postpartum Medicaid coverage as a policy strategy to combat postpartum morbidity and mortality and simultaneously improve child health.

\section{MEDICAID, PREGNANCY, AND POSTPARTUM CARE}

Medicaid is a government-funded health care coverage program for individuals with limited income or disabilities in the United States. It is jointly funded by the federal government and the states. Every state operates its individual Medicaid program within federal guidelines. States have flexibility in designing and administering their programs. As the nation's largest source of health care coverage with over 70 million beneficiaries, Medicaid has historically played a substantial role in providing maternity-related services for pregnant women, financing nearly half of all births in the United States. ${ }^{6}$ Per federal law, states are required to extend eligibility for pregnant women with incomes up to $138 \%$ of the federal poverty limit (FPL). Pregnancyrelated coverage lasts through 60 days postpartum while the infant is eligible for coverage through the first year of life. Services generally covered for women include prenatal care, childbirth, family planning, and delivery services although states have the discretion to delineate specific maternity care benefits under Medicaid.

With increasing maternal mortality rates and preterm births rising, disproportionately among racial and ethnic minority women nationally, ${ }^{7,8}$ there is increasing concern among clinicians, public health advocates, and policymakers that current Medicaid postpartum coverage policies are insufficient to ensure the health of mothers and infants. ${ }^{6}$ Beyond the 60 -day postpartum Medicaid coverage, mothers may still experience medical vulnerability and maternal mortality due to a myriad of factors including child-birth complications, pain, underlying chronic conditions, depression, and anxiety all in the context of adverse social determinants of health. In some states, $>50 \%$ of pregnancy-associated deaths occur between 43 and 364 days postpartum. ${ }^{8}$ As demonstrated in the study by Liu et al., the psychological impacts of COVID-19 likely amplify the stressors that postpartum women must navigate while caring for a young infant. These factors have led to increasing recognition that the clinical scope of postpartum care clearly exceeds 60 days. $^{9}$ Under current state policies, postpartum women can continue to qualify for Medicaid after 60 days, particularly in states with Medicaid expansion. However, Medicaid income eligibility levels for general adults in states without

\footnotetext{
${ }^{1}$ Center for Child Health Policy and Advocacy, Baylor College of Medicine, Houston, TX, USA. ${ }^{2}$ Children's Hospital Los Angeles, Department of Pediatrics, Keck School of Medicine
} of USC, Los Angeles, CA, USA. ${ }^{凶}$ email: Raphael@bcm.edu

Received: 15 November 2021 Revised: 12 December 2021 Accepted: 14 December 2021

Published online: 1 February 2022 
expansion are much more stringent than for pregnant women. ${ }^{9}$ Consequently, poor women may become uninsured 60 days postpartum because their income is too high to qualify for Medicaid.

\section{STATE-LEVEL POLICY INITIATIVES TO EXTEND POSTPARTUM CARE UNDER MEDICAID}

Recognizing that continuous coverage for low-income women after pregnancy has the potential to improve maternal and infant outcomes, states have implemented a diverse set of policies to address the loss of coverage. ${ }^{8}$ Medicaid expansion broadens coverage for postpartum women with incomes up to $138 \% \mathrm{FPL}$ able to retain coverage beyond 60 days postpartum. An added benefit is that the federal government pays $90 \%$ of the cost for the expansion population. ${ }^{9}$ Studies to date have demonstrated that Medicaid expansion is associated with decreased postpartum depression and lower maternal mortality rates. ${ }^{10,11}$ Other initiatives include raising parental income eligibility levels under Medicaid; extending pregnancy-related coverage beyond 60 days (e.g., 6 months) through Section 1115 waivers; expanding coverage for specific postpartum services (e.g., substance abuse) or specific populations (e.g., maternal mental health conditions); and postpartum coverage for family planning services. ${ }^{8,9}$ As of November 11, 2021, 26 states have Medicaid postpartum coverage extensions approved or pending as state action. ${ }^{12}$ While statelevel initiatives demonstrate the power of tailoring policy to the local level, the existence of such divergent strategies, and in some states, a paucity of any efforts highlights state-to-state variability of coverage experiences and persistent inequities in care.

\section{FEDERAL-LEVEL INITIATIVES TO BROADEN MEDICAID POSTPARTUM COVERAGE}

In recent years, several federal bills aimed at expanding Medicaid postpartum coverage to 12 months have progressed through Congress with varying success but ultimately not passed. These included the Helping Medicaid Offer Maternity Services (MOMS) Act of 2019, Patient Protection and Affordable Care Act Enhancement Act of 2020, and Black Maternal Health Momnibus. ${ }^{8}$ While these efforts have failed, the adverse economic impacts of the COVID-19 pandemic have elevated the urgency to address postpartum coverage expansion both in the short term and long term. In 2020, the Families First Coronavirus Response Act (FFCRA) provided states with enhanced federal matching funds if their Medicaid programs did not reduce eligibility standards and keep continuous enrollment for most members enrolled during the public health emergency (PHE). ${ }^{9}$ The continuous enrollment requirement will expire at the end of the month in which the PHE ends, currently expected to occur in 2022. Upon expiration of the PHE, a substantial percentage of postpartum women will become uninsured and become subject to pre-pandemic redetermination and renewal processes for Medicaid.

Building on the FFCRA, the American Rescue Plan Act of 2021 gives states an option to extend Medicaid postpartum coverage from 60 days to 12 months. ${ }^{6}$ States can extend coverage to a year by filing a State Plan Amendment to their Medicaid program. The new option takes effect in 2022 and would be available to states for 5 years. Given the impending PHE expiration and the time limits of the American Rescue Plan Act, more long-term federal strategies are needed. The proposed Build Back Better Act currently under negotiation in Congress outlines a number of policies to reform the nation's public coverage system. Principal among them is a requirement that all states extend full-benefit Medicaid postpartum coverage to one year after the end of pregnancy. This policy would build on the American Rescue Plan's state option by making the extension mandatory. States would receive their standard Medicaid matching rate for the extended coverage period. Despite the promise of a mandatory extension of postpartum coverage to a year, this provision exists within a large, aspirational piece of legislation likely to undergo intense and prolonged negotiations if even passed.

\section{CONCLUSIONS}

As policymakers debate the merits of the Build Back Better Act, urgent efforts are needed to define and implement permanent policy solutions to extend postpartum Medicaid coverage to a year. While not the sole solution to addressing maternal morbidity and mortality and poor maternal-infant bonding, Medicaid coverage represents a foundational element to supporting low-income families in complement with clinical and research initiatives, mitigation of structural racism, investments in community-based organizations, and development of a diverse perinatal workforce. ${ }^{6}$ To date, policy initiatives to extend postpartum Medicaid have been sporadic, variable in structure and implementation, and time-limited all with the potential to exacerbate inequities between states. ${ }^{9}$ With new legislative policies being considered, there is finally an opportunity to couple increasing awareness of optimal postpartum care with definitive and long-term action to support women and their families.

\section{REFERENCES}

1. Tully, K. P., Stuebe, A. M. \& Verbiest, S. B. The fourth trimester: a critical transition period with unmet maternal health needs. Am. J. Obstet. Gynecol. 217, 37-41 (2017).

2. Giallo, R., Woolhouse, H., Gartland, D., Hiscock, H. \& Brown, S. The emotionalbehavioural functioning of children exposed to maternal depressive symptoms across pregnancy and early childhood: a prospective Australian pregnancy cohort study. Eur. Child Adolesc. Psychiatry 24, 1233-1244 (2015).

3. Anderson, M. R., Salisbury, A. L., Uebelacker, L. A., Abrantes, A. M. \& Battle, C. L. Stress, coping and silver linings: how depressed perinatal women experienced the COVID-19 pandemic. J. Affect. Disord. 298, 329-336 (2022).

4. Wyszynski, D. F., Hernandez-Diaz, S., Gordon-Dseagu, V., Ramiro, N. \& Koenen, K. C. Stress levels among an international sample of pregnant and postpartum women during the COVID-19 pandemic. J. Matern. Fetal Neonatal Med. https:// doi.org/10.1080/14767058.2021.1936489 (2021).

5. Liu, C. H., Hyun, S., Mittal, L. \& Erdei, C. Psychological risks to mother-infant bonding during the COVID-19 pandemic. Pediatr. Res. https://doi.org/10.1038/ s41390-021-01751-9 (2021).

6. Moore, J. E., McLemore, M. R., Glenn, N. \& Zivin, K. Policy opportunities to improve prevention, diagnosis, and treatment of perinatal mental health conditions. Health Aff. 40, 1534-1542 (2021).

7. Creanga, A. A. et al. Pregnancy-related mortality in the United States, 2006-2010. Obstet. Gynecol. 125, 5-12 (2015).

8. Eckert, E. It's past time to provide continuous Medicaid coverage for one year postpartum. Health Aff. https://doi.org/10.1377/hblog20200203.639479 (2020).

9. Ranji, U., Gomez, I. \& Salganicoff, A. Expanding postpartum Medicaid coverage. Kaiser Family Foundation. https://www.kff.org/womens-health-policy/issue-brief/ expanding-postpartum-medicaid-coverage/ (2021).

10. Schuster, A. L. R. et al. The effect of the affordable care act on women's postpartum insurance and depression in 5 states that did not expand Medicaid, 2012-2015. Med. Care 60, 22-28 (2021).

11. Eliason, E. L. Adoption of Medicaid expansion is associated with lower maternal mortality. Women's Health Issues 30, 147-152 (2020).

12. Kaiser Family Foundation. Medicaid Postpartum Coverage Extension Tracker. https://www.kff.org/medicaid/issue-brief/medicaid-postpartum-coverageextension-tracker/ (2021).

\section{COMPETING INTERESTS}

The authors declare no competing interests.

\section{ADDITIONAL INFORMATION}

Correspondence and requests for materials should be addressed to Jean L. Raphael.

Reprints and permission information is available at http://www.nature.com/ reprints

Publisher's note Springer Nature remains neutral with regard to jurisdictional claims in published maps and institutional affiliations. 